\title{
Liver transplantation in patients with non-alcoholic steatohepatitis and alcohol-related liver disease: the dust is yet to settle
}

In the last two decades, non-alcoholic fatty liver disease (NAFLD), has transitioned from a non-existent entity to become one of the leading etiologies of chronic liver disease in the world. Its importance can be well inferred from its current estimated global prevalence which stands at $25.2 \%$ (1). With rising prevalence of adult obesity and diabetes mellitus (DM) among an aging population, NAFLD-related liver disease and mortality will continue to increase in the United States (2). NAFLD cases are forecasted to increase $21 \%$, from 83.1 million in 2015 to 100.9 million in 2030, while prevalent nonalcoholic steatohepatitis (NASH) cases will increase $63 \%$ from 16.52 to 27.00 million cases. Consequently, the incidence of decompensated cirrhosis is projected to increase by $168 \%$ to 105,430 cases by 2030 , hepatocellular carcinoma (HCC) by $137 \%$ to 12,240 cases, and liver deaths by $178 \%$ to an estimated 78,300 deaths in 2030 (2). NASH currently the second most common indication for waitlisted adults in the United States (3), and the leading indication for liver transplantation for woman in the United States (4). If the current rate continues, NAFLD will surpass other indications and become the leading cause of liver transplantation (LT) overall between 2020-2025 (5). A similar rise in the burden of alcohol-related liver disease (ALD) has been noted, leading to revisit into the ethical questions surrounding candidacy of LT in this groups of patients with many centers willing waive the strict 6-month sobriety rule for LT candidates.

The current series brings out the advances in NAFLD and ALD as it relates to liver transplantation, specifically focusing on the transplant candidates, issues related to wait listing, peri-transplant management, and their posttransplant outcomes.

NAFLD presents unique challenges in liver transplant candidates considering its association with metabolic syndrome, and increased prevalence of CAD (6). Agbim et al. has diligently discussed evaluation of liver transplant candidates with NASH. Patients with NASH cirrhosis has an increased thrombotic risk, leading to higher rates of pre-transplant portal vein thrombosis (PVT) than other causes of cirrhosis. Studies have shown worse outcomes in liver transplant recipients with PVT (7). De Leeuw et al. have discussed implication of PVT in NASH patients, and potential preventative strategies (8). Obesity plays a vital role in selection of liver transplant candidates as it affects post-transplant outcomes (9). There is a paucity of data on acute-on-chronic liver failure (ACLF) in patients with NASH cirrhosis. Using National Inpatient Sample data, a recent study has shown that NASH cirrhosis is the most rapidly growing indication for ACLF-related hospitalization and use of hospital resources in the United State (10). Using data from the United Network for Organ Sharing (UNOS) registry from 2005-2017 Sundaram et al. have further confirmed NAFLD being the fastest rising etiology of cirrhosis associated with ACLF among patients listed in the US. It is expected that as the NAFLD population continues to grow and age, patients with NAFLD-ACLF will likely have the highest risk of waitlist mortality (11). The inciting factors such as a higher rate of infections and circulatory failure, metabolic derangements such as obesity and diabetes which plays potentially key roles in driving the pathophysiology, clinical course, and prognosis of NASH patients with ACLF has been extensively reviewed by Doycheva and Thuluvath (12).

Bariatric surgery is currently the most effective management of morbid obesity and has been offered to patients both in the pre- and post-LT setting. The techniques attempted in LT recipients most commonly include sleeve gastrectomy, gastric bypass surgery with few cases of gastric banding and biliopancreatic diversion. However, there is lack of evidencebased data on the optimal management for patients with obesity and who are liver transplant candidates and/or recipients. Ahmed et al. have reviewed the evidence surrounding the role of bariatric surgery in the liver transplant patients and have reviewed outcomes with various forms of bariatric surgery (13). Another intriguing entity that has recently garnered increasin $\mathrm{g}$ attention is "Frailty" manifesting as sarcopenia which is an independent risk factor for mortality in cirrhosis, and several studies have recently shown poor outcomes following liver transplant with sarcopenia (4). Redman et al. have made a deeper dive into the pathophysiology of sarcopenia in patients with ALD and NAFLD, and have discussed implications of pretransplant sarcopenia in these groups of patients (14). Low-grade systemic inflammation, insulin resistance and atherogenic dyslipidemia are the key elements in the pathophysiology of NAFLD related renal dysfunction. Higher prevalence of chronic kidney disease (CKD) in NAFLD cirrhosis awaiting liver transplant leads to significantly higher requirement of simultaneous-liver-kidney transplant as compared to other etiologies. Maiwall et al. (15) have reviewed current literature 
surrounding pathogenetic basis of renal dysfunction in NAFLD and have recommended the choice of immunosuppression and use of intraoperative renal replacement therapy in context of intra and post-operative renal dysfunction in NASH transplant candidates. The high prevalence type 2 diabetes, obesity, metabolic syndrome, cardiovascular disease, and CKD in NAFLD patients makes them especially vulnerable group for worse post-transplant outcome which must be adequately managed during the peritransplant period for optimal graft outcomes. It is in this context Samji et al. (16) have put forward a comprehensive overview of the unique challenges these patients present in the peri-transplant period, and have discussed management approaches for best post-transplant outcomes. Maliakkal et al. have further discussed the cardiovascular outcomes in patients undergoing liver transplantations for NASH (17).

The implications of recurrent NASH on post LT outcomes, graft steatosis, progression to fibrosis, overall survival, and cardiovascular associations has been carefully reviewed by Taneja et al. (18). They have proposed potential preventative strategies, and extrapolated management strategies from non-transplant setting. Spiritos and Abdelmalek have further discussed the endogenous and exogenous drivers of post-transplant metabolic syndrome, role of chronic immunosuppression, and the prevalence and clinical significance of post-transplant metabolic syndrome in nonalcoholic steatohepatitis transplant recipients (19). They have also discussed potential management approach in this unique setting.

Recent studies have shown LT in the setting of ALD and its subset alcoholic hepatitis (AH) with improved survival rates even if transplanted with less than 6 months of sobriety (20). Shipley et al. (21) have discussed ethical and allocation-associated issues that arise when considering ALD and/or AH for LT. In addition, they have dissected into the history, controversies, current guidelines, and future directions of LT in this subgroup. In the background of emerging data on early LT in severe AH patients, Shipley et al. have reviewed the current status of LT in AH and highlighted the current challenges, barriers, and future prospects on this therapy for these sick patients with severe AH (21). While LT cures ALD, treatment of alcohol use disorder (AUD) must be included in the care plan to prevent a return to drinking and subsequent graft ALD. Shenoy et al. (22) emphasize that patients with underlying AUD must be recognized, offered brief interventions, and referred for multimodal multidisciplinary treatment that includes medications and psychotherapies along with sober support groups, family engagement, and a new dedication to healthy living in order to help sustain remission. Clearly such comprehensive care will increase LT candidacy in patients with ALD while optimizing clinical outcomes of patients transplanted with AUD. Additionally, post-transplant malignancy is emerging as an important cause of mortality in patients with cirrhosis undergoing liver transplant, and ALD predisposes them to a unique set of malignancies especially oropharyngeal and lung cancers. Evidences surrounding this association has been discussed in greater detail by Singh et al. (23).

In summary, the current series has quite comprehensively described several important concepts related to transplant candidacy, waitlist mortality, peri-transplant management, and posttransplant outcomes in patients with Non-Alcoholic Steatohepatitis and Alcohol Related Liver Disease with contribution from investigators across the world. As a guest editor, I sincerely hope that the reviews included in the current series will not only generate interest among the readers to learn but also will help inspire investigators with research ideas in search for the unknowns as the dust is yet to settle!

\section{Acknowledgments}

Funding: None.

\section{Footnote}

Provenance and Peer Review: This article was commissioned by the editorial office, Translational Gastroenterology and Hepatology for the series "Liver Transplantation in NASH and ALD". The article did not undergo external peer review.

Conflicts of Interest: All authors have completed the ICMJE uniform disclosure form (available at https://tgh.amegroups.com/ article/view/10.21037/tgh-2020-15/coif). The series "Liver Transplantation in NASH and ALD" was commissioned by the editorial office without any funding or sponsorship. All authors served as the unpaid Guest Editors of the series. SKS serves as an Editor-in-Chief of Translational Gastroenterology and Hepatology from November 2019 to October 2024. SKS reports grants from Gilead Sciences, Conatus Pharma, Intercept Pharma, Alexion, Genfit, Dova, Bayer, Exact Sciences, Shire NASH and 
Enanta; he also reports speaker's bureau from Intercept Pharma, Alexion and Dova, and he serves on the Advisory Board of Bayer, outside the submitted work. The authors have no other conflicts of interest to declare.

Ethical Statement: The authors are accountable for all aspects of the work in ensuring that questions related to the accuracy or integrity of any part of the work are appropriately investigated and resolved.

Open Access Statement: This is an Open Access article distributed in accordance with the Creative Commons AttributionNonCommercial-NoDerivs 4.0 International License (CC BY-NC-ND 4.0), which permits the non-commercial replication and distribution of the article with the strict proviso that no changes or edits are made and the original work is properly cited (including links to both the formal publication through the relevant DOI and the license). See: https://creativecommons.org/ licenses/by-nc-nd/4.0/.

\section{References}

1. Younossi ZM, Koenig AB, Abdelatif D, et al. Global epidemiology of nonalcoholic fatty liver disease-Meta-analytic assessment of prevalence, incidence, and outcomes. Hepatology 2016;64:73-84.

2. Estes C, Razavi H, Loomba R, et al. Modeling the epidemic of nonalcoholic fatty liver disease demonstrates an exponential increase in burden of disease. Hepatology 2018;67:123-33.

3. Wong RJ, Aguilar M, Cheung R, et al. Nonalcoholic steatohepatitis is the second leading etiology of liver disease among adults awaiting liver transplantation in the United States. Gastroenterology 2015;148:547-55.

4. Noureddin M, Vipani A, Bresee C, et al. NASH Leading Cause of Liver Transplant in Women: Updated Analysis of Indications For Liver Transplant and Ethnic and Gender Variances. Am J Gastroenterol 2018;113:1649-59.

5. Charlton MR, Burns JM, Pedersen RA, et al. Frequency and outcomes of liver transplantation for nonalcoholic steatohepatitis in the United States. Gastroenterology 2011;141:1249-53.

6. Kovalic AJ, Satapathy SK. The Role of Nonalcoholic Fatty Liver Disease on Cardiovascular Manifestations and Outcomes. Clin Liver Dis 2018;22:141-74.

7. Agbim U, Jiang Y, Kedia SK, et al. Impact of Nonmalignant Portal Vein Thrombosis in Transplant Recipients With Nonalcoholic Steatohepatitis. Liver Transpl 2019;25:68-78.

8. De Leeuw P, Agbim U. Pre-transplant portal vein thrombosis in non-alcoholic fatty liver disease patients — pathogenesis, risk factors, and implications on management. Transl Gastroenterol Hepatol 2022;7:27.

9. Satapathy SK, Jiang Y, Agbim U, et al. Posttransplant Outcome of Lean Compared With Obese Nonalcoholic Steatohepatitis in the United States: The Obesity Paradox. Liver Transpl 2020;26:68-79.

10. Axley P, Ahmed Z, Arora S, et al. NASH Is the Most Rapidly Growing Etiology for Acute-on-Chronic Liver Failure-Related Hospitalization and Disease Burden in the United States: A Population-Based Study. Liver Transpl 2019;25:695-705.

11. Sundaram V, Jalan R, Shah P, et al. Acute on chronic liver failure from nonalcoholic fatty liver disease: a growing and aging cohort with rising mortality. Hepatology 2021;73:1932-44.

12. Doycheva I, Thuluvath PJ. Acute-on-chronic liver failure in liver transplant candidates with non-alcoholic steatohepatitis. Transl Gastroenterol Hepatol 2020;5:38.

13. Ahmed Z, Khan MA, Vazquez-Montesino LM, et al. Bariatric surgery, obesity and liver transplantation. Transl Gastroenterol Hepatol 2022;7:25.

14. Redman JS, Kaspar M, Puri P. Implications of pre-transplant sarcopenia and frailty in patients with non-alcoholic steatohepatitis and alcoholic liver disease. Transl Gastroenterol Hepatol 2022;7:29.

15. Maiwall R, Gupta M. Peri-transplant renal dysfunction in patients with non-alcoholic steatohepatitis undergoing liver transplantation. Transl Gastroenterol Hepatol 2020;5:18.

16. Samji NS, Heda R, Satapathy SK. Peri-transplant management of nonalcoholic fatty liver disease in liver transplant candidates Transl Gastroenterol Hepatol 2020;5:10.

17. Maliakkal BJ. Pathogenesis of non-alcoholic fatty liver disease and implications on cardiovascular outcomes in liver transplantation. Transl Gastroenterol Hepatol 2020;5:36. 
18. Taneja S, Roy A. Nonalcoholic steatohepatitis recurrence after liver transplant. Transl Gastroenterol Hepatol $2020 ; 5: 24$.

19. Spiritos Z, Abdelmalek MF. Metabolic syndrome following liver transplantation in nonalcoholic steatohepatitis. Transl Gastroenterol Hepatol 2021;6:13.

20. American Diabetes Association. Classification and Diagnosis of Diabetes: Standards of Medical Care in Diabetes-2018. Diabetes Care 2018;41:S13-27.

21. Shipley LC, Singal AK. Liver transplantation for alcoholic hepatitis. Transl Gastroenterol Hepatol 2020;5:26.

22. Shenoy A, Salajegheh A, Shen NT. Multimodal multidisciplinary management of alcohol use disorder in liver transplant candidates and recipients. Transl Gastroenterol Hepatol 2022;7:28.

23. Singh A, De A, Singh V. Post-transplant malignancies in alcoholic liver disease. Transl Gastroenterol Hepatol 2020;5:30.

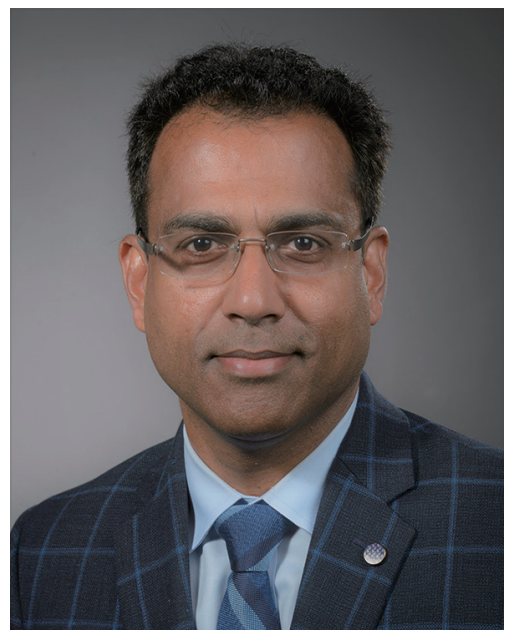

Sanjaya K. Satapathy

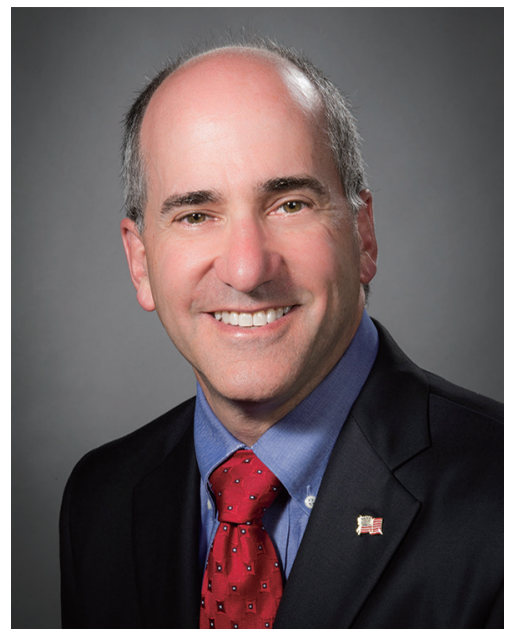

David E. Bernstein

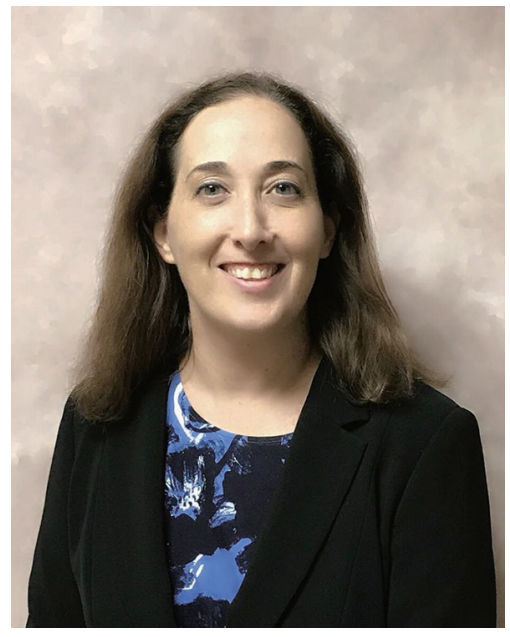

Nitzan C. Roth

Sanjaya K. Satapathy ${ }^{1,2}$, MBBS, MD, DM, MS (Epidemiology), FACG, AGAF, FASGE, FAASLD

(Email: ssatapat@northwell.edu)

David E. Bernstein ${ }^{3,4}$, MD, FAASLD, FACG, FACP, AGAF

(Email:dbernste@nortbwell.edu)

Nitzan C. Roth ${ }^{1,2}$, MD, PhD

(Email: nroth2@nortbwell.edu)

${ }^{1}$ Division of Hepatology at Sandra Atlas Bass Center for Liver Diseases \& Transplantation, Manhasset, NY, USA;

${ }^{2}$ Department of Medicine, Barbara and Zucker School of Medicine/Northwell Health, Manhasset, NY, USA;

${ }^{3}$ Division of Hepatology and Sandra Atlas Bass Center for Liver Diseases, Northwell Health, Hempstead, NY, USA;

${ }^{4}$ Donald and Barbara Zucker School of Medicine at Hofstra/Northwell, Northwell Health, Hempstead, NY, USA

Received: 29 September 2020; Accepted: 23 December 2020; Published: 25 July 2022.

doi: $10.21037 /$ tgh-2020-15

View this article at: http://dx.doi.org/10.21037/tgh-2020-15

doi: $10.21037 / \operatorname{tgh}-2020-15$

Cite this article as: Satapathy SK, Bernstein DE, Roth NC. Liver transplantation in patients with non-alcoholic steatohepatitis and alcohol-related liver disease: the dust is yet to settle. Transl Gastroenterol Hepatol 2022;7:23. 